\title{
Noninvasive Measurement of Skin Autofluorescence Is Increased in Patients with Systemic Sclerosis: An Indicator of Increased Advanced Glycation Endproducts?
}

\author{
ANDREA K. MURRAY, TONIA L. MOORE, JOANNE B. MANNING, CHRISTOPHER E.M. GRIFFITHS, \\ and ARIANE L. HERRICK
}

ABSTRACT. Objective. Skin autofluorescence noninvasively assesses expression of advanced glycation endproducts and therefore potentially the presence of oxidative stress that is implicated in the pathogenesis of systemic sclerosis (SSc). We investigated whether autofluorescence was increased in patients with SSc, primary Raynaud's phenomenon (RP), and morphea as compared to healthy controls.

Methods. Measurements of autofluorescence were made at 5 upper limb sites in 16 healthy controls, 16 patients with diffuse cutaneous SSc (dcSSc), 15 with limited cutaneous SSc (lcSSc), 15 with primary $\mathrm{RP}$, and 13 with morphea. For patients with morphea, additional measurements were made at the affected and an adjacent unaffected site.

Results. Autofluorescence was significantly increased in patients with dcSSc but not lcSSc as compared to controls at the proximal phalanx [dcSSc median 0.15, interquartile range (IQR) $0.10-0.24$, vs control 0.10, IQR 0.07-0.13; p = 0.014], dorsum of the hand (dcSSc 0.17, IQR 0.11-0.36, vs control 0.12, IQR 0.09-0.17; $\mathrm{p}=0.031$ ), the wrist (dcSSc 0.22, IQR 0.13-0.33, vs control 0.13, IQR 0.09-0.18; $\mathrm{p}=$ 0.005 ), and forearm (dcSSc 0.19 , IQR $0.12-0.47$, vs control 0.14 , IQR $0.10-0.16 ; p=0.022$ ). There was a trend for autofluorescence to be increased in patients with lcSSc and at morphea sites, compared to noninvolved skin.

Conclusion. Autofluorescence is increased in patients with dcSSc compared to primary RP and to healthy controls. This suggests increased oxidative stress and the potential for autofluorescence as a biomarker. (First Release July 1 2012; J Rheumatol 2012;39:1654-8; doi:10.3899/jrheum.111359)

Key Indexing Terms:

SYSTEMIC SCLEROSIS

RAYNAUD'S PHENOMENON

MORPHEA

ADVANCED GLYCATION ENDPRODUCTS

OXIDATIVE STRESS

From the Musculoskeletal Research Group, School of Translational Medicine, and the Dermatology Centre, the University of Manchester, Manchester Academic Health Science Centre, Salford Royal National Health Service (NHS) Foundation Trust, Salford; Photon Science Institute, University of Manchester, Manchester, UK

Supported by the Raynaud's and Scleroderma Association (HP14). A.K. Murray was supported by a University of Manchester Stepping Stone Award.

A.K. Murray, Musculoskeletal Research Group, School of Translational Medicine, The University of Manchester, Manchester Academic Health Science Centre, Salford Royal NHS Foundation Trust, and Photon Science Institute, University of Manchester; T.L. Moore, Musculoskeletal Research Group, School of Translational Medicine, The University of Manchester, Manchester Academic Health Science Centre, Salford Royal NHS Foundation Trust; J.B. Manning, Salford Royal NHS Foundation Trust; C.E.M. Griffiths, Dermatology Centre, University of Manchester, Manchester Academic Health Science Centre, Salford Royal NHS Foundation Trust; A.L. Herrick, Musculoskeletal Research Group, School of Translational Medicine, The University of Manchester, Manchester Academic Health Science Centre, Salford Royal NHS Foundation Trust. Address correspondence to Dr. A.K. Murray, Musculoskeletal Research Group, School of Translational Medicine, Salford Royal NHS Foundation, Manchester M68HD,UK.E-mail: Andrea.murray@manchester.ac.uk Accepted for publication March 23, 2012.
Systemic sclerosis ( $\mathrm{SSc}$ ) is a multisystem connective tissue disease, characterized by fibrosis and vascular abnormalities, for which at present there is no effective treatment ${ }^{1}$. SSc is subtyped into diffuse cutaneous ( $\mathrm{dcSSc}$ ) or limited cutaneous (lcSSc) disease on the basis of the extent of skin involvement ${ }^{2}$.

Although it is likely that oxidative stress, mediated by free radicals, contributes to SSc pathogenesis ${ }^{3,4,5,6}$ with expression of nitrotyrosine (a measure of free radical damage) being increased in forearm skin of patients with $\mathrm{SSc}^{7}$, its role has been relatively little studied because of the difficulty in noninvasive measurement. Advanced glycation endproducts (AGE) are compounds formed during oxidative stress that are known to be associated with microvascular changes, specifically damage and inflammation. Increased circulating levels of $\mathrm{AGE}^{8}$ and increased expression of AGE in skin biopsies ${ }^{9}$ have been reported in patients with SSc.

In this pilot study we investigated skin fluorescence, a noninvasive method of assessing the presence of AGE and of 
quantifying oxidative damage and stress (validated in patients with diabetes ${ }^{10,11,12,13}$ ) as a tool for measuring free radical injury in SSc. We tested the hypothesis that noninvasive measurement would identify differences in AGE levels in skin between patients with SSc, primary Raynaud's phenomenon (RP; idiopathic episodic color change of fingers and toes), and morphea (localized scleroderma) and healthy control subjects. Upper limbs were chosen for investigation because extremities are usually the site of first and maximal skin thickening in SSc (and in patients with lcSSc the extremities are the only sites of skin thickening), and because RP (both primary and secondary to $\mathrm{SSc}$ ) is usually more marked in the upper than the lower extremities. Patients with primary RP were included in order to increase understanding as to why they, in contrast to those with SSc, do not progress to irreversible tissue injury. Knowing whether AGE levels are increased in primary RP, and how these levels differ from those in SSc, would contribute to our understanding of the pathogenesis of primary RP and SSc. Patients with morphea were included in order to increase understanding of the differences between the pathogenesis of the skin changes in SSc and those in morphea, and to elucidate why the changes in morphea remain confined to the skin and underlying tissues, and do not progress to involve internal organs ${ }^{14}$.

\section{MATERIALS AND METHODS}

Sixteen patients with dcSSc, 15 with lcSSc, 15 with primary RP, and 13 with morphea and 16 healthy controls were recruited into the study. Demographic data are displayed in Table 1. All were white except for 1 who was Asian. All patients gave written consent and the study was approved by Salford and
Trafford Research Ethics Committee. All participants were asked to be caffeine- and nicotine-free for 4 hours prior to the study.

Following acclimatization in a dimly lighted, temperature-controlled laboratory at $23^{\circ} \mathrm{C}$ for $20 \mathrm{~min}$, an image of the nailfold of the ring finger of the nondominant hand was taken as described ${ }^{15}$. Nailfold capillaroscopy was performed to determine the relationship between increased fluorescence and microvascular disease severity.

Measurements of skin autofluorescence were made using a spectrometer (USB400 UV-Vis; Ocean Optics, Dunedin, FL, USA) and bifurcated fiberoptic probe (1000 $\mu \mathrm{m} \mathrm{UV/vis).} \mathrm{The} \mathrm{bifurcated} \mathrm{probe} \mathrm{delivered} \mathrm{ultraviolet}$ (UV) light from the LED source (372-383 nm, full-width half-maximum) to the skin and collected both the reflected UV light and autofluorescence emitted from the skin (spectrum shown in Figure 1), returning them to the spectrometer. Relative skin autofluorescence was calculated as the ratio of fluorescence emission intensity $(430-700 \mathrm{~nm})$ divided by the UV reflection intensity $(350-430 \mathrm{~nm})$ to account for any fluctuations in incident UV light levels. Measurements of skin autofluorescence were made at 5 upper limb sites: distal and proximal phalanges (of the nondominant ring finger), hand, wrist, and forearm (all dorsal aspect, nondominant limb). For patients with morphea, additional measurements were made at the affected site and an adjacent unaffected site. Each measurement was taken twice and the mean of the ratio calculated. To assess reproducibility, 4 healthy control participants made a repeat visit within 3 weeks, during which skin was not expected to change.

Nailfold capillaroscopy images were analyzed using semiautomated software, as described ${ }^{16}$, to measure the following features of the capillaries: semiautomated intercapillary distance, capillary width, tortuosity (curliness), and derangement (variance in direction). Values for these measurements are arbitrary at present, because of the way that they are calculated within the program. Capillaroscopy was carried out on only 1 finger due to time constraints (ideally all nailfolds would have been examined).

Skin autofluorescence data were found to be skewed by inspection and were therefore log-transformed. Differences between measurements at different sites and between groups were analyzed with a general linear model with age as a cofactor. Differences between skin affected and unaffected by mor-

Table 1. Demographics, nailfold capillaroscopy results, and autofluorescence data (ratio of autofluorescence/reflected ultraviolet light) in patients with diffuse cutaneous systemic sclerosis (dcSSc), limited cutaneous systemic sclerosis (lcSSc), primary Raynaud's phenomenon (PRP), morphea, and healthy controls [results are median (interquartile range)].

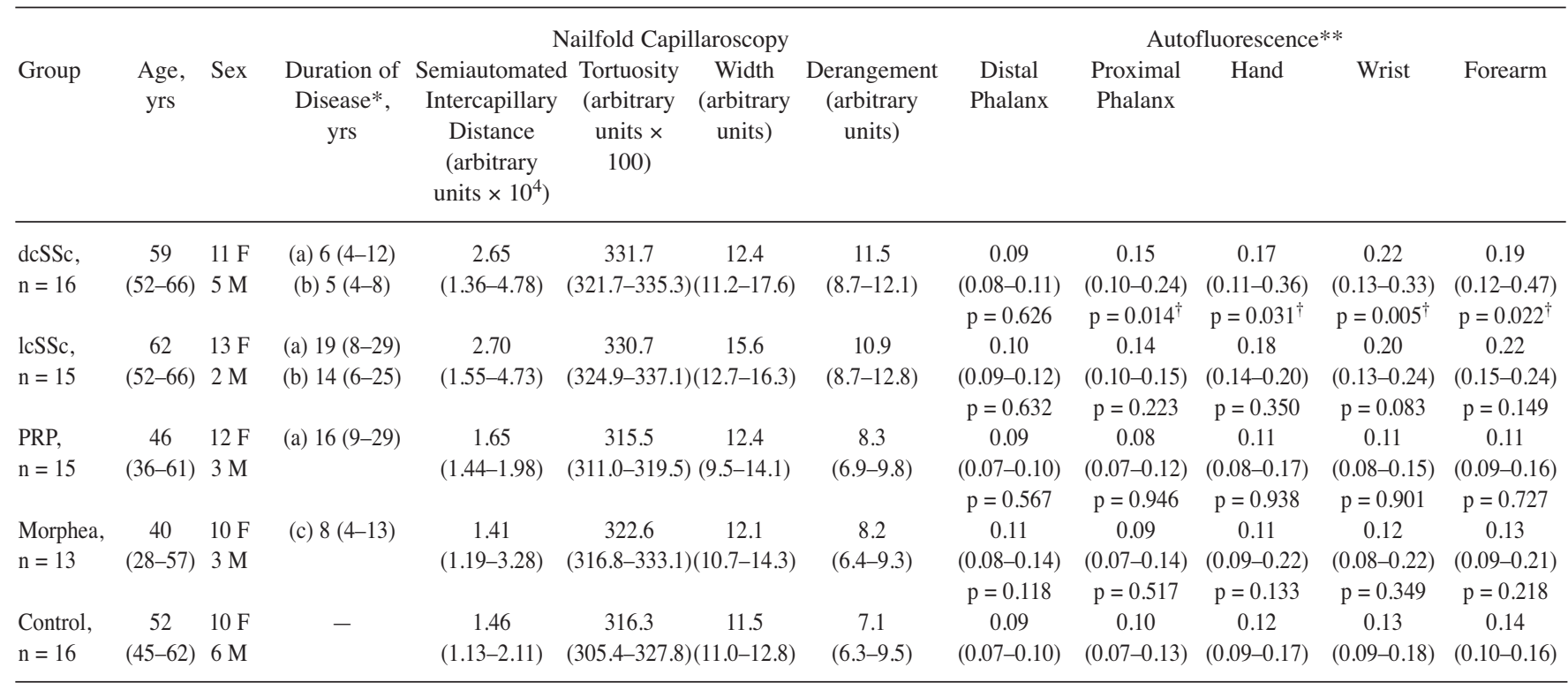

* (a) Raynaud's phenomenon; (b) duration of SSc from first non-Raynaud's clinical feature; (c) duration of morphea from first noted changes in skin. ** $\mathrm{p}$ value is difference from control group; ${ }^{\dagger} \mathrm{p}<0.05$. 


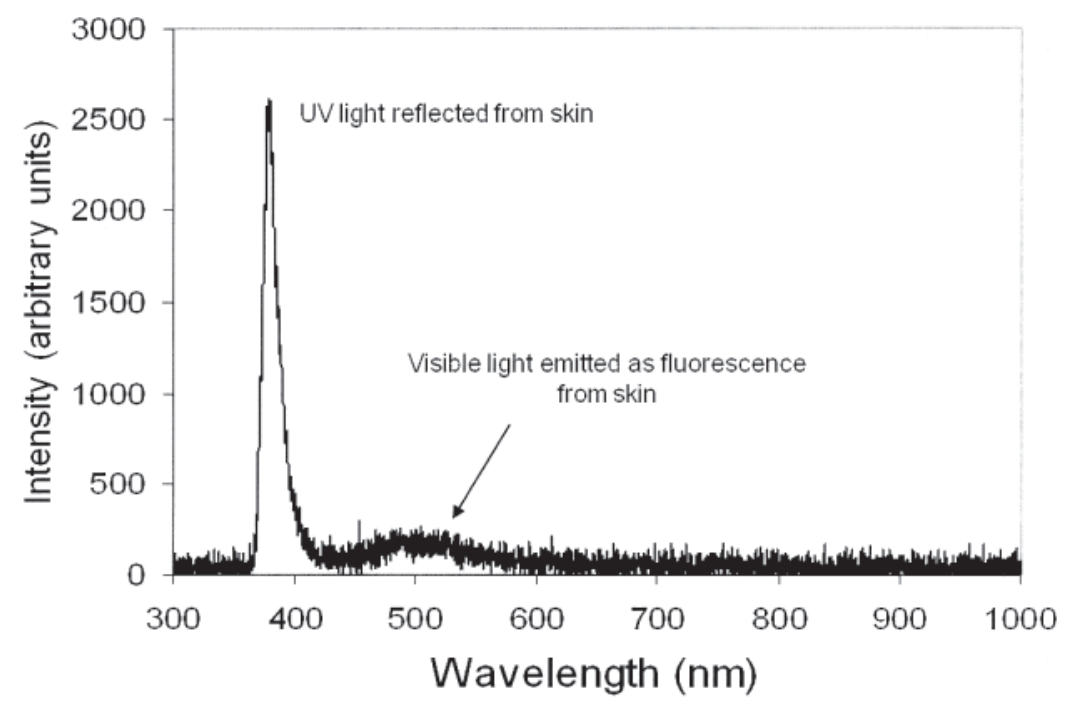

Figure 1. Typical spectrum of skin autofluorescence: left hand peak is the reflected ultraviolet (UV) light from the skin and the right hand (smaller peak) is the fluorescence emitted from the skin.

phea were analyzed using a t test. Correlations for all groups combined (75 subjects) between fluorescence intensity and nailfold capillary features were examined using Pearson's correlation coefficient. Reproducibility was assessed in 2 ways: for immediate repeat measurement, using the 2 measurements taken at each upper limb site for all participants, and in 4 subjects for repeat measurements taken several days apart, using data from repeat visits. Data were examined in a Bland-Altman analysis ${ }^{17}$. Analysis was carried out in SPSS v15 (SPSS Inc., Chicago, IL, USA) and Stata v10 (StataCorp LP, College Station, TX, USA).

\section{RESULTS}

Between-group comparisons of autofluorescence. Data for autofluorescence measurements are presented in Table 1 and Figure 2 ( $\mathrm{p}$ values in Table 1 are age-adjusted; data in Figure 2 are log-transformed). Skin autofluorescence was significantly increased in patients with dcSSc (compared to controls) at the proximal phalanx, dorsum of the hand, wrist, and forearm. Patients with lcSSc did show an increased level of fluorescence, but these levels did not reach significance with respect to controls. Patients with primary RP or morphea did not show an increased level of fluorescence compared to controls.

Although autofluorescence was increased at sites affected by morphea [median 0.13 , interquartile range (IQR) $0.10-0.19]$ as compared to adjacent unaffected sites $(0.11$, IQR $0.10-0.15)$, this difference was not statistically significant.

Correlations between autofluorescence and microvascular structure (assessed by capillaroscopy). Values for nailfold capillaroscopy are shown in Table 1. Correlations (taken for all subject groups together) were as follows: proximal phalanx with tortuosity, $\mathrm{r}=0.238, \mathrm{p}=0.049$; hand with distance, $\mathrm{r}=$ $0.272, \mathrm{p}=0.024$; hand with tortuosity, $\mathrm{r}=0.257, \mathrm{p}=0.033$; wrist with distance, $\mathrm{r}=0.398, \mathrm{p}=0.001$; wrist with tortuosity, $\mathrm{r}=0.398, \mathrm{p}=0.001$; forearm with distance, $\mathrm{r}=0.408, \mathrm{p}<$ 0.001 ; and forearm with tortuosity, $r=0.357, p=0.003$. No correlations were found for capillary width or derangement.
Reproducibility. Reproducibility was assessed using the Bland-Altman approach ${ }^{17}$, which considers the size of difference between pairs of measurements across the range of observed values. Reproducibility as assessed using the 2 immediately repeated measurements had $95 \%$ limits of agreement (i.e., $95 \%$ of differences between measurements would be expected to lie within this range) of -0.09 to 0.09 , with an intraclass correlation coefficient (ICC; a measure of linear association between the 2 scores, where ICC $=1$ would imply a perfect linear association) of 0.89 and a repeatability of 0.09 (below which the difference would lie with $95 \%$ probability) for alpha equal to 0.05 . Reproducibility for the mean of the 2 measurements taken at each repeat visit had $95 \%$ limits of agreement of -0.07 to 0.12 , ICC of 0.66 , and repeatability of 0.11 .

\section{DISCUSSION}

Our results demonstrate an increase in skin fluorescence in patients with dcSSc. This may be indicative of the presence of increased levels of AGE, which would implicate the contribution of free radicals to the SSc disease process. This has implications for management, lending further support for research into new lines of therapy, for example with antioxidants. We have also demonstrated the feasibility of skin autofluorescence (a validated, noninvasive test for oxidative stress and free radical involvement) as an important tool in studies of pathophysiology of SSc and in monitoring of the treatment response. That autofluorescence was higher in patients with $\mathrm{dcSSc}$ than lcSSc most likely reflects the more extensive skin involvement in those with diffuse cutaneous disease.

Our finding that autofluorescence intensity correlated with the degree of microvascular abnormality (as measured by capillaroscopy) is consistent with microvascular disease driving oxidative stress. Conversely, autofluorescence intensity was 


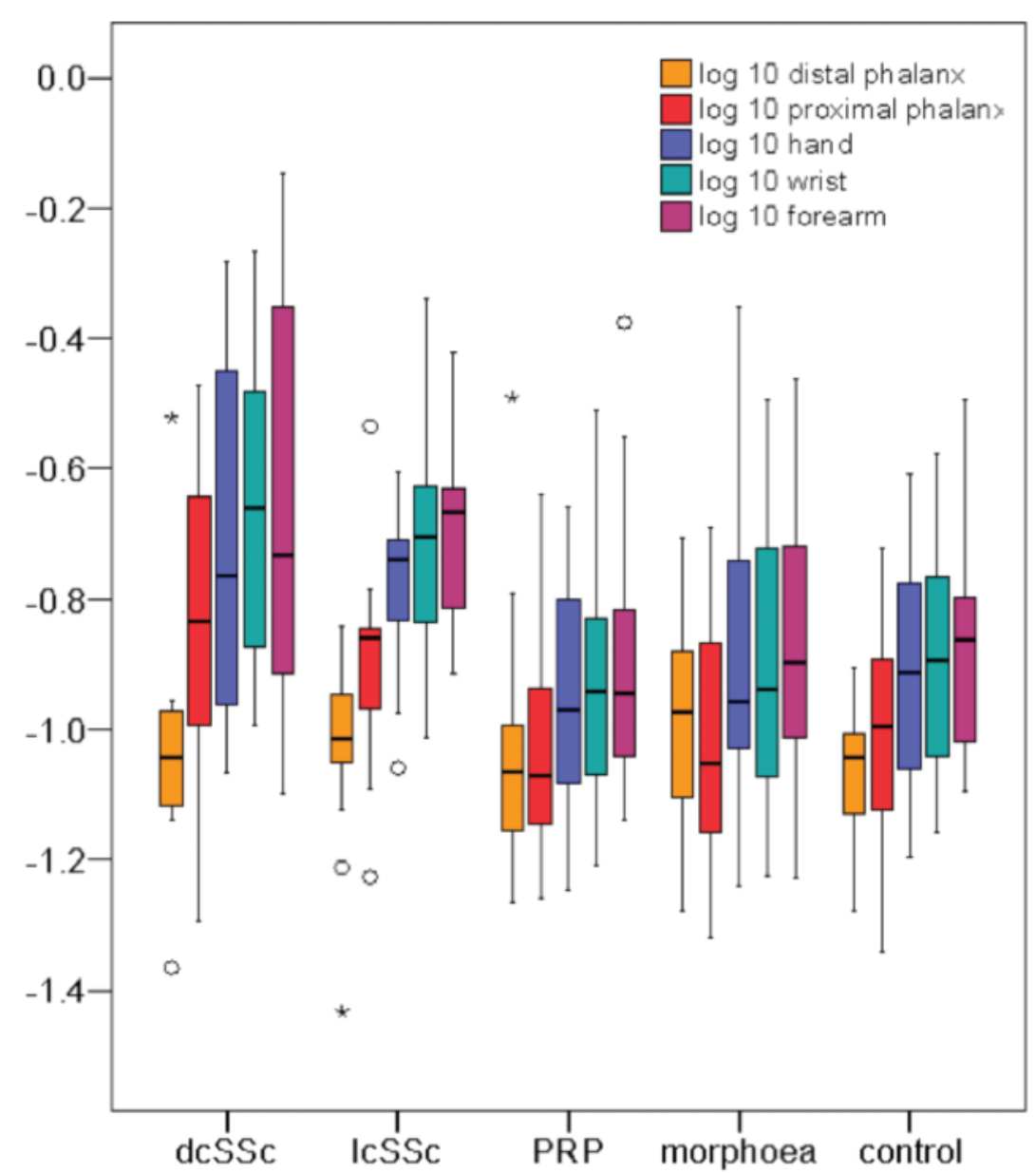

Figure 2. Box plots of autofluorescence intensity/UV reflection ratio (log-transformed) for subject groups and sites (distal phalanx, proximal phalanx, hand, wrist, forearm). Diffuse cutaneous systemic sclerosis (dcSSc); limited cutaneous systemic sclerosis (lcSSc); and primary Raynaud's phenomenon (PRP). Box plots represent median (central horizontal bar) and interquartile range (IQR; lower and upper limits of boxes) of sample values. Whiskers extend to the minimum and maximum values in the sample, except where outlying values are individually indicated by circles (outliers, IQR 1.5-3) or asterisks (extreme values $>3 \mathrm{IQR}$ ).

similar in healthy controls and patients with primary RP, who have either no change or only very subtle structural microvascular change. The trend for increased autofluorescence in morphea plaques was of interest: the pathogenesis of morphea has been underresearched and studies involving larger numbers of patients and including more patients with early disease would be of interest.

We confirmed for both patients and healthy controls the variation in fluorescence intensity across different anatomical $\operatorname{sites}^{18}$, highlighting the importance of establishing an optimum location for measurements to be made longitudinally. In healthy controls, skin fluorescence is known to be associated with aging ${ }^{19,20}$, and therefore age-matched controls should be included where possible.

Alternative causes for increases in skin fluorescence are discussed by Mulder, et $a l^{12}$ and include fluorescent interstitial and intracapillary compounds that might be expected to be increased in patients with SSc, due to the increased capillary permeability observed in this patient cohort ${ }^{21}$. The reason for no increase in fluorescence at the distal phalanx in patients with dcSSc remains unexplained. However, there may be other mechanisms in finger skin that cause a compensatory decrease in fluorescence, and if so, these need to be further understood if fluorescence is to be used as a measure of oxidative stress in future studies. The most likely explanations for the skin not autofluorescing as intensely as expected in the fingers of patients with SSc are skin structure (e.g., skin thickening) and differences in skin pigmentation. Skin pigmentary changes are well recognized in SSc, although their cause is unknown. We did not collect data on the degree of skin pigmentation. Skin thickening and changes in skin color may both cause higher absorption of incident excitation light, thus decreasing the amount available to cause fluorescence. Therefore skin thickening, which in patients with SSc is usu-

Personal non-commercial use only. The Journal of Rheumatology Copyright (c) 2012. All rights reserved. 
ally most marked in the fingers, may have "masked" the anticipated increased autofluorescence in the fingers.

Increased skin fluorescence indicates that levels of advanced glycation endproducts are increased in patients with $\mathrm{dcSSc}$, providing further evidence that oxidative stress is a part of the disease process and suggesting that autofluorescence may serve as a biomarker for disease. Further studies are required to investigate the relationships between autofluorescence, skin thickening, and skin pigmentation.

\section{ACKNOWLEDGMENT}

We thank Andy Vail, Health Sciences Research Group, Manchester Academic Health Science Centre, The University of Manchester, Salford Royal Hospital, Salford, UK, for statistical advice.

\section{REFERENCES}

1. Ramos-Casals M, Fonollosa-Pla V, Brito-Zerón P, Siso-Almirall A. Targeted therapy for systemic sclerosis: How close are we? Nat Rev Rheumatol 2010;6:269-78.

2. LeRoy EC, Black C, Fleischmajer R, Jablonska S, Krieg T, Medsger TA Jr, et al. Scleroderma (systemic sclerosis): Classification, subsets and pathogenesis. J Rheumatol 1988;15:202-5.

3. Herrick AL, Matucci Cerinic M. The emerging problem of oxidative stress and the role of antioxidants in systemic sclerosis. Clin Exp Rheumatol 2001;19:4-8.

4. Herrick AL, Rieley F, Schofield D, Hollis S, Braganza JM, Jayson MI. Micronutrient antioxidant status in patients with primary Raynaud's phenomenon and systemic sclerosis. J Rheumatol 1994;21:1477-83.

5. Bruckdorfer KR, Hillary JB, Bunce T, Vancheeswaran R, Black $\mathrm{CM}$. Increased susceptibility to oxidation of low-density lipoproteins isolated from patients with systemic sclerosis. Arthritis Rheum 1995;38:1060-7.

6. Ogawa F, Shimizu K, Muroi E, Hara T, Hasegawa M, Takehara K, et al. Serum levels of 8-isoprostane, a marker of oxidative stress, are elevated in patients with systemic sclerosis. Rheumatology 2006;45:815-8.

7. Cotton SA, Herrick AL, Jayson MI, Freemont AJ. Endothelial expression of nitric oxide synthases and nitrotyrosine in systemic sclerosis skin. J Pathol 1999;189:273-8.

8. Kaloudi O, Basta G, Perfetto F, Bartoli F, Del Rosso A, Miniati I, et al. Circulating levels of $\mathrm{N}$ epsilon-(carboxymethyl) lysine are increased in systemic sclerosis. Rheumatology 2007;46:412-6.
9. Davies C, Herrick AL, Cordingley L, Freemont AJ, Jeziorska M. Expression of advanced glycation end products (AGE) and their receptor (RAGE) in skin from patients with systemic sclerosis. Rheumatology 2009;48:876-82.

10. Gerrits EG, Lutgers HL, Kleefstra N, Graaff R, Groenier KH, Smit AJ, et al. Skin autofluorescence: A tool to identify type 2 diabetic patients at risk for developing microvascular complications. Diabetes Care 2008;31:517-21.

11. Lutgers HL, Graaff R, Links TP, Ubink-Veltmaat LJ, Bilo HJ, Gans $\mathrm{RO}$, et al. Skin autofluorescence as a non-invasive marker of vascular damage in patients with type 2 diabetes. Diabetes Care 2006;29:2654-9.

12. Mulder DJ, Water TV, Lutgers HL, Graaff R, Gans RO, Zijlstra F, et al. Skin autofluorescence, a novel marker for glycemic and oxidative stress-derived advanced glycation endproducts: An overview of current clinical studies, evidence, and limitations. Diabetes Technol Ther 2006;8:523-35.

13. Meerwaldt R, Lutgers HL, Links TP, Graaff R, Baynes JW, Gans $\mathrm{RO}$, et al. Skin autofluorescence is a strong predictor of cardiac mortality in diabetes. Diabetes Care 2007;30:107-12.

14. Laxer RM, Zulian F. Localized scleroderma. Curr Opin Rheumatol 2006;18:606-13.

15. Anderson ME, Allen PD, Moore T, Hillier V, Taylor CJ, Herrick AL. Computerized nailfold video capillaroscopy - A new tool for assessment of Raynaud's phenomenon. J Rheumatol 2005;32:841-8.

16. Murray AK, Feng K, Moore TL, Allen PD, Taylor CJ, Herrick AL. Preliminary clinical evaluation of semi-automated nailfold capillaroscopy in the assessment of patients with Raynaud's phenomenon. Microcirculation 2011;18:440-7.

17. Bland JM, Altman DG. Statistical methods for assessing agreement between two methods of clinical measurement. Lancet 1986;i:307-10.

18. Na R, Stender IM, Ma L, Wulf HC. Autofluorescence spectrum of skin: Component bands and body site variations. Skin Res Technol 2000;6:112-7

19. Beisswenger PJ, Howell S, Mackenzie T, Corstjens H, Muizzuddin $\mathrm{N}$, Matsui MS. Two fluorescent wavelengths, 440(ex)/520(em) nm and $370(\mathrm{ex}) / 440(\mathrm{em}) \mathrm{nm}$, reflect advanced glycation and oxidation end products in human skin without diabetes. Diabetes Technol Ther 2012;14:285-92.

20. Na R, Stender IM, Henriksen M, Wulf HC. Autofluorescence of human skin is age-related after correction for skin pigmentation and redness. J Invest Dermatol 2001;116:536-40.

21. Herrick AL. Vascular function in systemic sclerosis. Curr Opin Rheumatol 2000;12:527-33. 